\title{
Expression of miR-296-5p as predictive marker for radiotherapy resistance in early-stage laryngeal carcinoma
}

\author{
Danielle Maia ${ }^{1}$, Ana Carolina de Carvalho ${ }^{1,2}$, Maria Aderuza Horst ${ }^{1}$, André Lopes Carvalho ${ }^{2,3}$, \\ Cristovam Scapulatempo-Neto ${ }^{4}$ and Andre Luiz Vettore ${ }^{1,5^{*}}$
}

\begin{abstract}
Purpose: Definitive radiation therapy is the mainstay of treatment for early stage laryngeal squamous cell carcinoma (LSCC). However, up to 30\% of the patients do not respond to radiotherapy. Unfortunately, we are unable to predict which tumors are likely to respond to radiation, and which will be resistant and persist. Therefore, the development of novel markers to predict response to radiotherapy is urgently needed. This study was designed to evaluate the expression pattern of microRNAs (miRNAs) in LSCC in order to identify markers capable of segregating radioresistant and radiosensitive tumors and to investigate the relationship between the expression of these miRNAs and the prognosis of LSCC.
\end{abstract}

Methods: The expression profile of 667 miRNAs was determined in an initial screening of nine early-stage LSCC samples (5 radioresistant and 4 radiosensitive) using TaqMan Low-Density Array (TLDA). Real-time polymerase chain reactions were performed to validate the expression of selected miRNAs in an expanded LSCC cohort (20 radioresistant and 14 radiosensitive). The miRNA expression level was scored as high or low based on the median of the expression in the LSCC samples.

Results: A comprehensive miRNA expression profiling enabled the identification of four miRNAs (miR-296-5p miR452, miR-183* and miR-200C) differentially expressed in radioresistant LSCC. Moreover, the analysis of additional 34 LSCC samples, confirmed the expression of miR-296-5p as significantly related to radioresistance $(p=0.002)$ as well as an association of this marker with recurrence $(p=0.025)$ in early stage laryngeal cancer.

Conclusions: This study indicates that miR-296-5p expression is associated with resistance to radiotherapy and tumor recurrence in early stage LSCC, showing the feasibility of this marker as a novel prognostic factor for this malignance. Furthermore, miR-296-5p expression could be helpful in the identification of tumors resistant to radiotherapy; thus aiding the clinicians in the choice of the best therapeutic scheme to be used in each case.

Keywords: Early-stage laryngeal cancers, Molecular marker, MicroRNAs, Radioresistance, miR-296-5p

\section{Background}

Laryngeal cancer represents the second most common site of head and neck tumors [1,2], and has squamous cell carcinoma as the most important histology [2]. Accounting for approximately 160,000 new cases per

\footnotetext{
*Correspondence: andre.vettore@gmail.com

${ }^{5}$ Cancer and Stem Cell Biology Program, Duke-NUS Graduate Medical

School, Singapore, Singapore

Full list of author information is available at the end of the article
}

year and for $2.5 \%$ of all tumors in males, LSCC remains a major disease burden worldwide. Treatment options comprise surgery, radiotherapy, chemotherapy or a combination of modalities [3, 4]. Despite refinement of multimodal therapies, the 5-year relative survival percentage has been stable at around 60 for the last decades for early laryngeal cancer [5].

Definitive radiation therapy is the mainstay of treatment for early stage (T1-T2 N0) laryngeal squamous cell carcinoma (LSCC). However, $6-15 \%$ of patients with 
stage 1 disease and $20-31 \%$ of patients with stage 2 disease do not respond to radiotherapy [6]. If a patient fails radiotherapy, surgery is the main treatment option that may offer a cure, and up to $50 \%$ will require a total laryngectomy as salvage treatment. Moreover, when used as salvage surgery after a failed radiotherapy course, laryngectomy presents an increased complication rate [7]. Remarkably, for these failed cases, definitive cancer cure is delayed by the course of radiotherapy with a risk of tumor progression, adversely affecting patient prognosis still further. Unfortunately, we are unable to predict which tumors are likely to respond to radiation, and those, which will be resistant and persist. Therefore, it would be desirable to find novel and valuable markers to predict beforehand which patients will benefit from radiotherapy.

Relevant clinical factors associated with local recurrence after radiotherapy are tumor size, tumor stage, overall treatment time and radiotherapy fraction size [8-10]. Treatment choice is now mainly based on T-stage [11], however, this is insufficient to warrant recurrencefree survival as observed in heterogeneous responses and survival rates presented by LSCC patients [6].

Molecular markers have the potential to help in the selection of treatment through response prediction and determination of cancer prognosis $[12,13]$ Previous studies have identified few molecular markers, such as p53, cyclin D1, EGFR, VEGF, IGF1R, COX2 and Ki67, associated with local relapse and overall survival in early laryngeal carcinoma [12, 14-16]. However, some of these findings are conflicting and none of these markers are being used in clinical practice.

MicroRNAs (miRNAs) are non-coding RNAs with 18-25 nucleotides, described as negative regulators of gene expression in a variety of multicellular organisms. These small molecules harbor the capacity to bind and silence specific messenger RNA (mRNA) targets, inducing their degradation, or inhibiting their translation [17]. MicroRNAs play important roles in various biological processes, such as apoptosis, cell proliferation, cell differentiation, tumorigenesis, and multidrug resistance (MDR) [18]. Emerging evidences revealed that miRNA expression patterns are significantly changed in cancer cells submitted to ionizing radiation [19]. Many studies have shown modulation of radiosensitivity by altering miRNA levels in various malignances, such as lung cancer cells in vitro [20] and breast cancer cells in vivo [21].

The purposes of this study were to assess the global miRNA expression profile of patients with T1-T2 N0 laryngeal cancer treated with primary radiation therapy, in order to identify miRNAs able to segregate radioresistant and radiosensitive tumors, thus serving as markers to predict radiotherapy resistance. Moreover, the role of
miRNAs in prognosis will be assessed by searching for associations between the expression of selected miRNAs and clinical and pathological parameters of LSCC patients.

\section{Methods}

\section{Patients and tissue samples}

This retrospective study included 34 patients with primary LSCC tumors (glottic and supraglottic) treated with radiotherapy as first and single modality treatment with curative intent at the Barretos Cancer Hospital, Barretos, São Paulo, Brazil, between 2000 and 2010. Diagnosis of LSCC was determined according to the WHO criteria.

Patients were identified as harboring radioresistant or radiosensitive tumors depending upon their response to radiotherapy. Tumors were staged according to the TNM classification [22] with all cases being clinically $\mathrm{T} 1-\mathrm{T} 2$, clinically nodal negative (N0) and negative for distant metastases (M0) at the time of treatment.

The radioresistant cohort consisted of 20 LSCC patients. The criteria for radioresistant tumors were: (a) radiotherapy had to be given as a single modality treatment with curative intent for a biopsy-proven squamous cell carcinoma of the larynx; (b) biopsy-proven recurrent squamous cell carcinoma, with the recurrence occurring at the local or locoregional original anatomical site after finishing the course of radiotherapy.

The radiosensitive cohort of tumors was comprised of 14 LSCC patients. The criteria for radiosensitive tumors were: (a) radiotherapy had to be given as a single modality treatment with curative intent for a biopsy-proven squamous cell carcinoma of the larynx; (b) post treatment, patients had a minimum follow-up of 3 years with no evidence of a recurrent laryngeal tumor. The definition for LSCC recurrence adopted in this study is the presence of tumor cell in the same site of the primary tumor or radiological evidence of locoregional disease after finishing the course of radiotherapy and during the first 3 years of follow-up.

This study was approved by the local IRB for the use of archival biopsy material and patient clinical data collection. Medical records of patients were reviewed for standard demographic data, pretreatment classification, tumor staging, radiation therapy parameters, and disease outcome.

\section{Radiotherapy}

Radiation treatment parameters were as follows: bilateral laryngeal opposed wedged fields using beam energies of $6 \mathrm{MV}, 5$ fractions per week, without planned treatment breaks. Patients were treated with daily fractions of 2-2.25 Gy to a total median dose of 66 Gy (range 49.579 Gy) over 47 days (range 38-79 days). 


\section{RNA isolation and CDNA synthesis}

Biopsies sections (5- $\mu \mathrm{m}$ thick) were performed from pretreatment archival tissue blocks of the radioresistant and radiosensitive tumors. Total RNA was isolated using the miRNeasy FFPE Kit (Qiagen, Hilden, Germany), according to the manufacturer's instructions. Ten nanograms of total RNA from each sample were subjected to reverse transcription using the TaqMan MicroRNA Reverse Transcription Kit (Applied Biosystems, Foster City, CA, USA) and specific stem-loop primers for each of the microRNAs selected, in accordance with the manufacturer's recommendations.

\section{Global microRNA profiling}

Global microRNA expression profiling of FFPE samples in a discovering set comprised of 9 patients (5 from the radioresistant group and 4 from the radiosensitive group) was performed using the TaqMan Human MicroRNA Cards Set v2.0 (Applied Biosystems), allowing the evaluation of the expression level of 667 human microRNAs. Forty nanograms of total RNA from each sample was reverse transcribed into cDNA using the TaqMan miRNA Reverse Transcription Kit (Applied Biosystems) and Megaplex RT Primers (Applied Biosystems). Next, the product obtained from the RT reactions was preamplified using the TaqMan PreAmp Master Mix Kit (Applied Biosystems) and Megaplex PreAmp Primers (Applied Biosystems). The amplified-cDNA was then transferred to the TaqMan Human MicroRNA Cards Set v2.0 and the amplification was carried out in the 7900HT Real-Time PCR System (Applied Biosystems). The data obtained was analyzed using the software DataAssist v3.0 (Applied Biosystems). The fold-change difference between radioresistant and radiosensitive cases was calculated using the $2^{-\Delta \Delta C t}$ method [23]. The small nuclear RNA U6 was used as endogenous control and the radiosensitive cases were assigned as reference. Cases were scored as differentially expressed if a 4-fold-change increase was observed.

\section{Validation of the differentially expressed microRNAs}

The expression level of the miRNAs selected for the validation step was evaluated in a total of 34 samples $(20$ radioresistant and 14 radiosensitive) using individual TaqMan MicroRNA Assays (Applied Biosystems). Each assay was conducted using the TaqMan MicroRNA Reverse Transcription kit (Applied Biosystems) according to the manufacturer's protocols. Briefly, $10 \mathrm{ng}$ of total RNA were reverse-transcribed using MultiScribe Reverse Transcriptase (Applied Biosystems) and a stemloop primer (Applied Biosystems). The mixture was incubated at $16^{\circ} \mathrm{C}$ for $30 \mathrm{~min}, 42^{\circ} \mathrm{C}$ for $30 \mathrm{~min}$ and $85^{\circ} \mathrm{C}$ for 5 min. Quantitative RT-PCR (qRT-PCR) was performed using TaqMan PCR kit (Applied Biosystems) on a 7500 Fast Real-Time PCR System (Applied Biosystems). Three technical replicates of each sample were performed for every microRNA. To evaluate the differential expression of each microRNA between radioresistant and radiosensitive cases, the $2^{-\Delta \Delta \mathrm{Ct}}$ method was employed [23]. Mean Ct values of $U 6$ small nuclear RNA was used for normalization.

\section{Statistical analysis}

To search for differentially expressed microRNAs between both groups in the global miRNA expression profiling, $\Delta \mathrm{Ct}$ values from each microRNA were evaluated using the t-Student test with the BenjaminiHochberg adjustment for false discovery rate (FDR) as implemented in the DataAssist software v3.0 (Applied Biosystems). The individual assay results were analyzed after normalization of data. $\triangle \mathrm{CT}$ values of microRNAs assayed in the validation step using individual TaqMan assays were used for comparisons between groups using Mann-Whitney U test for non-normal distribution.

The miRNA levels measured during the validation step were converted into discrete variables by splitting the samples into two classes (high and low expression) using the $\Delta \mathrm{Ct}$ median level considering all samples evaluated as cutoff. The Chi square test and Fisher's exact test were used to evaluate the associations between miRNA expression and clinical variables, as appropriate. The Kaplan-Meier method was used to estimate disease-free survival (DFS) of patients, and the log-rank test was used to examine the differences between groups. The DFS was defined as the time interval between the date of the end of the radiation therapy and the date of diagnosis of the first recurrence, or last date of follow-up if recurrence was not observed. A p value of $<0.05$ indicated the presence of statistically significant difference between groups. All statistical analyses were performed using SPSS statistics 20.0.

\section{Results \\ Characteristics of the patients}

The clinical and histological characteristics of the 34 patients enrolled in this study are listed in Table 1. Patients were mainly male (88.2\%), with age ranging from 39 to 85 years (median 62.5 years). Tobacco use (current or former) was reported by $91.2 \%$ of the patients. Primary tumor sites were predominantly glottic (79.4\%) and tumor stage at diagnosis was $\mathrm{T} 1$ in $47.1 \%$ and $\mathrm{T} 2$ in $52.9 \%$ of the cases. Most patients (70.6\%) received at least 70 Gy in a range from 6,300 to $7,020 \mathrm{cGy}$ (total median dose, $66 \mathrm{~Gy}$ ), within a median delivery interval of treatment of 55 days (range 38-79 days). None of the clinical and therapeutic variables were correlated with the radioresistant tumors, as noted in Table 1. 
Table 1 Clinical and pathological data of the patients enrolled in the study

\begin{tabular}{|c|c|c|c|c|}
\hline & $\begin{array}{l}\text { Number } \\
\text { of cases (\%) }\end{array}$ & $\begin{array}{l}\text { Radiosensi- } \\
\text { tive } \\
\text { n (\%) }\end{array}$ & $\begin{array}{l}\text { Radioresist- } \\
\text { ant } \\
\text { n(\%) }\end{array}$ & $X^{2}$ ( $p$ value) \\
\hline \multicolumn{5}{|l|}{ Gender } \\
\hline Male & $30(88.2)$ & $12(40.0)$ & $18(60.0)$ & 1.0 \\
\hline Female & $4(11.8)$ & $2(50.0)$ & $2(50.0)$ & \\
\hline \multicolumn{5}{|l|}{ Age } \\
\hline$\leq 60$ years & $16(47.1)$ & $5(31.2)$ & $11(68.8)$ & 0.51 \\
\hline$>60$ years & $18(52.9)$ & $9(50.0)$ & $9(50.0)$ & \\
\hline \multicolumn{5}{|c|}{ Tobacco consumption } \\
\hline Yes & $31(91.2)$ & $14(45.2)$ & $17(54.8)$ & 0.25 \\
\hline No & $3(8.8)$ & $0(0)$ & $3(100.0)$ & \\
\hline \multicolumn{5}{|l|}{ Tumor site } \\
\hline Supraglottic & 7 (20.6) & $2(28.6)$ & $5(71.4)$ & 1.0 \\
\hline Glottic & $27(79.4)$ & $12(44.4)$ & $15(55.6)$ & \\
\hline \multicolumn{5}{|l|}{ Tumor stage } \\
\hline । & $16(47.1)$ & $8(50)$ & $8(50)$ & 0.49 \\
\hline$\|$ & $18(52.9)$ & $6(33.3)$ & $12(66.7)$ & \\
\hline \multicolumn{5}{|c|}{ Radiotherapy dose } \\
\hline$<70 \mathrm{~Gy}$ & $10(29.4)$ & $5(50.0)$ & $5(50.0)$ & 0.7 \\
\hline$\geq 70 \mathrm{~Gy}$ & $24(70.6)$ & $9(37.5)$ & $15(62.5)$ & \\
\hline \multicolumn{5}{|l|}{ Treatment time } \\
\hline$\leq 55$ days & $20(58.8)$ & $9(45.0)$ & $11(55.0)$ & 0.72 \\
\hline$>55$ days & $14(41.2)$ & $5(37.5)$ & $9(64.3)$ & \\
\hline
\end{tabular}

\section{MicroRNA expression in LSCC samples}

Comprehensive miRNA profiles were generated from LSCC samples collected from radioresistant $(n=5)$ and radiosensitive $(\mathrm{n}=4)$ patients using a quantitative RTPCR array platform (Additional file 1: Table S1). miRNAs were considered differentially expressed in concordance with two criteria: (1) miRNAs with FDR-adjusted p value $<0.3$, as previously suggested [24], and (2) an increase in the expression level of the radioresistant group in comparison to the radiosensitive group greater than 4-fold. Thus, by using a FDR-adjusted $\mathrm{p}$ value $<0.3,42$ miRNAs could be considered as differentially expressed between radioresistant and radiosensitive samples considering a 4-fold difference. From those, based in the expression level and literature searching, 15 miRNA were selected for further analysis. All miRNAs that were at least 2-fold up-regulated or down-regulated are presented in Table 2.

Due to the scarcity of RNA quantity in many samples, it would be impossible to evaluate all 15 differentially expressed miRNAs selected in all samples. After a deep review of the available literature data from the $15 \mathrm{miR}$ NAs identified in the discovery series, miR-452, miR200c, miR-183* and miR-296-5p were selected for further analyses. The expression levels of these four selected miRNAs were determined in the entire cohort of samples (20 radioresistant and 14 radiosensitive) and, by using the median value as threshold cutoff, the miRNA expression was scored as low level, below the median value, and high level, above the median value (Additional file 2: Table S2).

\section{miR-296-5p expression is associated with patient prognosis}

The expression levels (high or low) of miR-452, miR200c, miR-183* and miR-296-5p were analyzed for potential correlations with clinical characteristics of the LSCC patients, including age, gender, tobacco consumption, tumor site, tumor stage, and radiosensitivity. The high expression of miR-296-5p showed a significant correlation with resistance to radiotherapy $(\mathrm{p}=0.010$; Table 3$)$. No associations were observed between the clinical features and expression status of the other miRNAs tested.

The comparison of the expression of miR-296-5p between the radioresistant and radiosensitive groups reveals a significant higher expression of this miRNA in the radioresistant group ( $\mathrm{p}=0.002$, Fig. 1). Expression of miR-452, miR-200c and miR-183* between the radioresistant and radiosensitive groups were not significant associated with radioresistance (Fig. 1).

The 3-year disease-free survival (DFS) rate considering all patients included in the study was $34 \%$ (Fig. 2a). Additionally, we investigated the association between the expression levels of the four selected miRNAs and the 3 -year DFS. According to this analysis, $41.6 \%$ of the patients with low expression of miR-296-5p presented recurrences, while relapses were detected in $82.4 \%$ of the LSCC patients with high expression of this miRNA, and this difference was statistically significant $(\mathrm{p}=0.025$, $\mathrm{OR}=8.6$, 95 CI 1.7-42.2, Fig. 2b). Expression levels of miR-452, miR-200c and miR-183* were not associated with disease-free survival in this cohort (data not shown).

\section{Discussion}

Despite recent advances in the multidisciplinary management of early stage laryngeal cancer, including surgical resection or larynx-preservation protocols, a substantial proportion of patients with localized or locally advanced disease will eventually relapse and die [25]. Radiotherapy as the initial treatment is commonly used, offering a possibility to preserve laryngeal function and reserving surgery as a salvage procedure. Nodal involvement, gender, tumor volume, impaired vocal cord mobility, tumor invasion of cartilage, and overall treatment time are factors found to be of prognostic importance. However, these prognostic factors fail to differentiate patients with good outcome of radiotherapy from those patients that will 
Table 2 A list of miRNAs differentially expressed according to the TaqMan Human MicroRNA Array

\begin{tabular}{|c|c|c|c|c|c|}
\hline \multicolumn{3}{|l|}{ Up-regulated } & \multicolumn{3}{|l|}{ Down-regulated } \\
\hline microRNA & Fold-change & FDR-adjusted p-value & microRNA & Fold-change & FDR-adjusted $p$ value \\
\hline hsa-miR-642 & 2.02 & 0.3869 & hsa-miR-106b & 2.01 & 0.2162 \\
\hline hsa-miR-744 & 2.02 & 0.4254 & hsa-miR-302b & 2.05 & 0.5657 \\
\hline hsa-miR-221 & 2.03 & 0.3475 & hsa-miR-136* & 2.06 & 0.5306 \\
\hline hsa-miR-193b* & 2.07 & 0.3359 & hsa-miR-127-5p & 2.09 & 0.5626 \\
\hline hsa-miR-193b & 2.08 & 0.4149 & hsa-miR-152 & 2.09 & 0.3678 \\
\hline hsa-miR-18b & 2.11 & 0.3012 & hsa-miR-34c-5p & 2.09 & 0.4936 \\
\hline hsa-miR-222*- & 2.15 & 0.4874 & hsa-miR-199a-5p & 2.11 & 0.6033 \\
\hline hsa-miR-944 & 2.16 & 0.4246 & hsa-miR-190b & 2.12 & 0.6416 \\
\hline hsa-miR-766- & 2.21 & 0.3723 & hsa-miR-140-3p & 2.14 & 0.4081 \\
\hline hsa-miR-106b* & 2.22 & 0.3155 & hsa-let-7i* & 2.16 & 0.5682 \\
\hline hsa-miR-942 & 2.35 & 0.3275 & hsa-miR-195 & 2.17 & 0.508 \\
\hline hsa-miR-135a & 2.41 & 0.1506 & hsa-miR-302c & 2.17 & 0.5853 \\
\hline hsa-miR-652 & 2.41 & 0.2572 & hsa-miR-488 & 2.17 & 0.5469 \\
\hline hsa-miR-124 & 2.42 & 0.3872 & hsa-miR-411* & 2.17 & 0.5271 \\
\hline hsa-miR-105 & 2.51 & 0.2277 & hsa-miR-21 & 2.18 & 0.3333 \\
\hline hsa-miR-923 & 2.62 & 0.5362 & hsa-miR-590-5p & 2.18 & 0.37 \\
\hline hsa-miR-138 & 2.71 & 0.2162 & hsa-miR-29c & 2.23 & 0.4804 \\
\hline hsa-miR-181a-2* & 2.78 & 0.2034 & hsa-miR-454 & 2.24 & 0.3152 \\
\hline hsa-miR-23b & 2.84 & 0.2235 & hsa-miR-10b* & 2.25 & 0.5091 \\
\hline hsa-miR-224 & 2.85 & 0.2626 & hsa-miR-378* & 2.29 & 0.4961 \\
\hline hsa-miR-141 & 2.89 & 0.3926 & hsa-miR-380* & 2.31 & 0.4251 \\
\hline hsa-miR-205 & 3.13 & 0.2706 & hsa-miR-661 & 2.31 & 0.5051 \\
\hline hsa-miR-129-3p & 3.53 & 0.1788 & hsa-miR-10a & 2.34 & 0.1452 \\
\hline hsa-miR-188-5p & 3.58 & 0.5457 & hsa-miR-148b* & 2.34 & 0.4926 \\
\hline hsa-miR-107 & 3.81 & 0.1843 & hsa-miR-592 & 2.35 & 0.4694 \\
\hline hsa-miR-183 & 3.91 & 0.1201 & hsa-miR-449b & 2.36 & 0.526 \\
\hline hsa-miR-296-5p & 4.26 & 0.084 & hsa-miR-487a & 2.36 & 0.511 \\
\hline hsa-miR-23a & 4.58 & 0.1771 & hsa-miR-199a-3p & 2.37 & 0.4496 \\
\hline hsa-miR-22 & 4.78 & 0.3268 & hsa-miR-181a & 2.41 & 0.3499 \\
\hline hsa-miR-801 & 5.87 & 0.127 & hsa-miR-30a* & 2.48 & 0.3854 \\
\hline hsa-miR-200a & 6.22 & 0.3678 & hsa-miR-144* & 2.51 & 0.5976 \\
\hline hsa-miR-203 & 6.22 & 0.1755 & hsa-miR-509-3p & 2.56 & 0.4307 \\
\hline hsa-miR-429 & 6.68 & 0.1588 & hsa-miR-146b-5p & 2.57 & 0.224 \\
\hline hsa-miR-183* & 7.84 & 0.0203 & hsa-miR-154* & 2.57 & 0.3571 \\
\hline hsa-miR-200b* & 8.02 & 0.1744 & hsa-miR-638 & 2.61 & 0.4688 \\
\hline hsa-miR-601 & 8.31 & 0.2335 & hsa-miR-328 & 2.61 & 0.3059 \\
\hline hsa-miR-200b & 9.41 & 0.1332 & hsa-miR-656 & 2.62 & 0.4201 \\
\hline hsa-miR-200c & 9.77 & 0.1334 & hsa-miR-146b-3p & 2.68 & 0.328 \\
\hline hsa-miR-200a* & 9.86 & 0.0974 & hsa-miR-202 & 2.77 & 0.3485 \\
\hline hsa-miR-452 & 17.94 & 0.0238 & hsa-miR-548b-5p & 2.77 & 0.4288 \\
\hline hsa-miR-650 & 18.46 & 0.1454 & hsa-miR-340 & 2.78 & 0.2592 \\
\hline hsa-miR-378 & 28.01 & 0.3958 & hsa-miR-15a* & 2.81 & 0.2874 \\
\hline \multirow[t]{6}{*}{ hsa-miR-513-3p } & 229.63 & 0.355 & hsa-miR-29c* & 2.87 & 0.4847 \\
\hline & & & hsa-miR-29a* & 2.89 & 0.3125 \\
\hline & & & hsa-miR-132 & 2.91 & 0.2681 \\
\hline & & & hsa-miR-345 & 2.98 & 0.0946 \\
\hline & & & hsa-miR-214 & 3.02 & 0.4376 \\
\hline & & & hsa-miR-376a & 3.05 & 0.4487 \\
\hline
\end{tabular}


Table 2 continued

\begin{tabular}{|c|c|c|c|c|}
\hline \multicolumn{2}{|c|}{ Up-regulated } & \multicolumn{3}{|l|}{ Down-regulated } \\
\hline microRNA & Fold-change $\quad$ FDR-adjusted $p$-value & microRNA & Fold-change & FDR-adjusted $\mathrm{p}$ value \\
\hline & & hsa-miR-20a* & 3.11 & 0.183 \\
\hline & & hsa-miR-539 & 3.12 & 0.4698 \\
\hline & & hsa-miR-487b & 3.19 & 0.4356 \\
\hline & & hsa-miR-433 & 3.21 & 0.3942 \\
\hline & & hsa-miR-639 & 3.21 & 0.3717 \\
\hline & & hsa-miR-885-5p & 3.27 & 0.3696 \\
\hline & & hsa-miR-520c-3p & 3.28 & 0.3564 \\
\hline & & hsa-miR-127-3p & 3.29 & 0.4056 \\
\hline & & hsa-miR-99a* & 3.32 & 0.2645 \\
\hline & & hsa-miR-361-5p & 3.39 & 0.1345 \\
\hline & & hsa-miR-134 & 3.41 & 0.369 \\
\hline & & hsa-miR-130b* & 3.51 & 0.3143 \\
\hline & & hsa-miR-493 & 3.53 & 0.3315 \\
\hline & & hsa-miR-212 & 3.58 & 0.241 \\
\hline & & hsa-miR-19b-1* & 3.68 & 0.3595 \\
\hline & & hsa-miR-30d* & 3.74 & 0.091 \\
\hline & & hsa-miR-760 & 3.75 & 0.4218 \\
\hline & & hsa-miR-125b-1* & 3.77 & 0.3397 \\
\hline & & hsa-miR-140-5p & 3.81 & 0.1951 \\
\hline & & hsa-miR-34b* & 3.97 & 0.4022 \\
\hline & & hsa-miR-99b* & 4.17 & 0.214 \\
\hline & & hsa-miR-889 & 4.17 & 0.294 \\
\hline & & hsa-miR-516a & 4.17 & 0.244 \\
\hline & & hsa-miR-770 & 4.17 & 0.274 \\
\hline & & hsa-miR-671 & 4.55 & 0.174 \\
\hline & & hsa-miR-100 & 4.76 & 0.204 \\
\hline & & hsa-miR-543 & 4.76 & 0.264 \\
\hline & & hsa-miR-410 & 5.00 & 0.285 \\
\hline & & hsa-miR-495 & 5.00 & 0.385 \\
\hline & & hsa-miR-485 & 5.26 & 0.325 \\
\hline & & hsa-miR-411 & 5.56 & 0.235 \\
\hline & & hsa-miR-16-1* & 5.56 & 0.045 \\
\hline & & hsa-miR-382 & 5.88 & 0.345 \\
\hline & & hsa-miR-100* & 5.88 & 0.225 \\
\hline & & hsa-miR-7-1* & 5.88 & 0.505 \\
\hline & & hsa-miR-337 & 6.25 & 0.756 \\
\hline & & hsa-miR-451 & 6.25 & 0.236 \\
\hline & & hsa-miR-99a & 7.14 & 0.167 \\
\hline & & hsa-miR-218 & 7.69 & 0.847 \\
\hline & & hsa-miR-370 & 7.69 & 0.327 \\
\hline & & hsa-miR-379 & 8.33 & 0.788 \\
\hline & & hsa-miR-409 & 9.09 & 0.369 \\
\hline & & hsa-miR-376c & 10.00 & 0.1110 \\
\hline & & hsa-miR-432 & 11.11 & 0.2111 \\
\hline & & hsa-miR-214* & 12.50 & 0.1412 \\
\hline
\end{tabular}


Table 3 Correlation between the expression level of microRNAs miR-296-5p, miR-452, miR-183* and miR-200c and the clinical and pathological characteristics of the LSCC patients enrolled in the study

\begin{tabular}{|c|c|c|c|c|c|c|c|c|c|c|}
\hline \multirow[t]{2}{*}{ Variable } & \multirow[t]{2}{*}{ Categories } & \multirow[t]{2}{*}{ Number of cases } & \multicolumn{2}{|c|}{ miR-296-5p } & \multicolumn{2}{|l|}{ miR-452 } & \multicolumn{2}{|l|}{ miR-183* } & \multicolumn{2}{|c|}{ miR-200c } \\
\hline & & & $\begin{array}{l}\text { Low } \\
\text { n (\%) }\end{array}$ & $\begin{array}{l}\text { High } \\
\text { n (\%) }\end{array}$ & $\begin{array}{l}\text { Low } \\
\text { n (\%) }\end{array}$ & $\begin{array}{l}\text { High } \\
\text { n (\%) }\end{array}$ & $\begin{array}{l}\text { Low } \\
\text { n (\%) }\end{array}$ & $\begin{array}{l}\text { High } \\
\text { n (\%) }\end{array}$ & $\begin{array}{l}\text { Low } \\
\text { n (\%) }\end{array}$ & $\begin{array}{l}\text { High } \\
\text { n (\%) }\end{array}$ \\
\hline \multirow[t]{3}{*}{ Age (years) } & $\leq 60$ years & 16 & $6(35.3)$ & $10(58.8)$ & $8(47.1)$ & $8(47.1)$ & $8(47.1)$ & $8(47.1)$ & $8(50.0)$ & $8(47.1)$ \\
\hline & $>60$ years & 18 & $11(64.7)$ & $7(41.2)$ & $9(52.9)$ & $9(52.9)$ & $9(52.9)$ & $9(52.9)$ & $8(50.0)$ & $9(52.9)$ \\
\hline & $p(2$-sided $)$ & & NS & & NS & & NS & & NS & \\
\hline \multirow[t]{3}{*}{ Gender } & Female & 4 & $2(11.8)$ & $2(11.8)$ & $2(11.8)$ & $2(11.8)$ & $2(11.8)$ & $2(11.8)$ & $2(12.5)$ & $2(12.5)$ \\
\hline & Male & 30 & $15(88.2)$ & $15(88.2)$ & $15(88.2)$ & $15(88.2)$ & $15(88.2)$ & $15(88.2)$ & $14(87.5)$ & $15(88.2)$ \\
\hline & $p$ (2-sided) & & NS & & NS & & NS & & NS & \\
\hline \multirow[t]{3}{*}{ Smoking } & No & 3 & $0(0)$ & $3(17.6)$ & $1(5.9)$ & $2(11.8)$ & $0(0)$ & $3(17.6)$ & $2(12.5)$ & $1(9.1)$ \\
\hline & Yes & 31 & $17(100)$ & $14(82.4)$ & $16(94.1)$ & $15(88.2)$ & $17(100)$ & $14(82.4)$ & $14(87.5)$ & $16(94.1)$ \\
\hline & $p$ (2-sided $)$ & & NS & & NS & & NS & & NS & \\
\hline \multirow[t]{3}{*}{ Tumor site* } & Glottic & 27 & $15(88.2)$ & $12(70.6)$ & $14(82.4)$ & $13(76.5)$ & $14(82.4)$ & $13(76.5)$ & $14(87.5)$ & $12(70.6)$ \\
\hline & Supraglottic & 7 & $2(11.8)$ & $5(29.4)$ & $31(7.6)$ & $4(23.5)$ & $3(17.6)$ & $4(23.5)$ & $2(12.5)$ & $5(29.4)$ \\
\hline & $p$ (2-sided $)$ & & NS & & NS & & NS & & NS & \\
\hline \multirow[t]{3}{*}{ Tumor stage } & I & 16 & $7(41.2)$ & $9(52.9)$ & $9(52.9)$ & $7(41.2)$ & $7(41.2)$ & $9(52.9)$ & $7(43.8)$ & $9(52.9)$ \\
\hline & $\|$ & 18 & $10(58.8)$ & $8(47.1)$ & $8(47.1)$ & $10(58.8)$ & $10(58.8)$ & $8(47.1)$ & $9(56.2)$ & $8(47.1)$ \\
\hline & $p$ (2-sided $)$ & & NS & & NS & & NS & & NS & \\
\hline \multirow[t]{3}{*}{ Radiosensibility } & Sensivite & 14 & $11(64.7)$ & $3(17.6)$ & $8(47.1)$ & $6(35.3)$ & $9(52.9)$ & $5(29.4)$ & $7(43.8)$ & $7(41.2)$ \\
\hline & Resistant & 20 & $6(35.3)$ & $14(82.4)$ & $9(52.9)$ & $11(64.7)$ & $8(47.1)$ & $12(70.6)$ & $9(56.2)$ & $10(58.8)$ \\
\hline & $p$ (2-sided) & & 0.01 & & NS & & NS & & NS & \\
\hline
\end{tabular}

Italic value indicates statistical significance at $p<0.05$.

$p$ (Fisher's exact); NS ( $p>0.05)$.

not respond to the treatment. In spite of the high rates of response to radiotherapy, around 30\% of patients with small, good prognosis tumors are not cured by this treatment and must undergo salvage laryngectomy (partial or total). Therefore, an accurate and reproducible estimation of prognosis in patients with early laryngeal cancer is important as an opportunity to spare patients from ineffective and toxic therapies.

To better understand the biology of radiation response, analysis of the phenotype of tumor cells has been underway for the last several years. Previous studies have evaluated the prognostic significance of various biomarkers including angiogenesis markers, members of the proapoptotic family, cell cycle regulators, and proliferation markers in head and neck cancer [19, 20, 26-28]. However, results have been quite mixed, reflecting the multitude of factors that contribute to the complex tumor biology as well as the heterogeneity of laryngeal cancers in terms of biology, site, stage, and prognosis [20].

Several studies have reported specific miRNA expression in different cancer cell types and in distinct differentiation tumor stages [21, 29]. Some of them have exploited the potential of miRNAs as prognostic markers in HNSCC and found associations between the low levels of miRNAs such as miR-205 and let-7d and high levels of miR-451 with disease progression and increased risk for local and regional recurrence [30]. As diagnostic markers, the ratio between the expression of miR-221 and miR-375 was able to discriminate HNSCC and normal samples with a specificity of $93 \%$ and a sensitivity of 92\% [31]. Moreover, although a small number of cases was evaluated, Fletcher et al. found a specific expression of miR-205 in metastatic lymph nodes of HNSCC patients [32].

Specifically in laryngeal cancer, five miRNAs (miR331-3p, miR 603, miR, 1303, miR-660-5p and miR212-3p) were identified specifically in plasma samples from affected patients, while theis expression was absent in the normal population. Therefore, these findings suggest miRNAs as promissor biomarkers for laryngeal cancer early diagnostic [33]. Cao et al. identified that miR-21, miR-93, miR-205, and miR-708 were upregulated and miR-125b and miR-145 were downregulated between adjacent normal tissue and laryngeal cancer, these results points to the importance of miRNAs in tumorigenesis and tumor progression of laryngeal cancer [34].

In turn, some studies have exploited the potential role of miRNAs in modulating radiosensitivity in different tumors. Given the fact that the local control of 

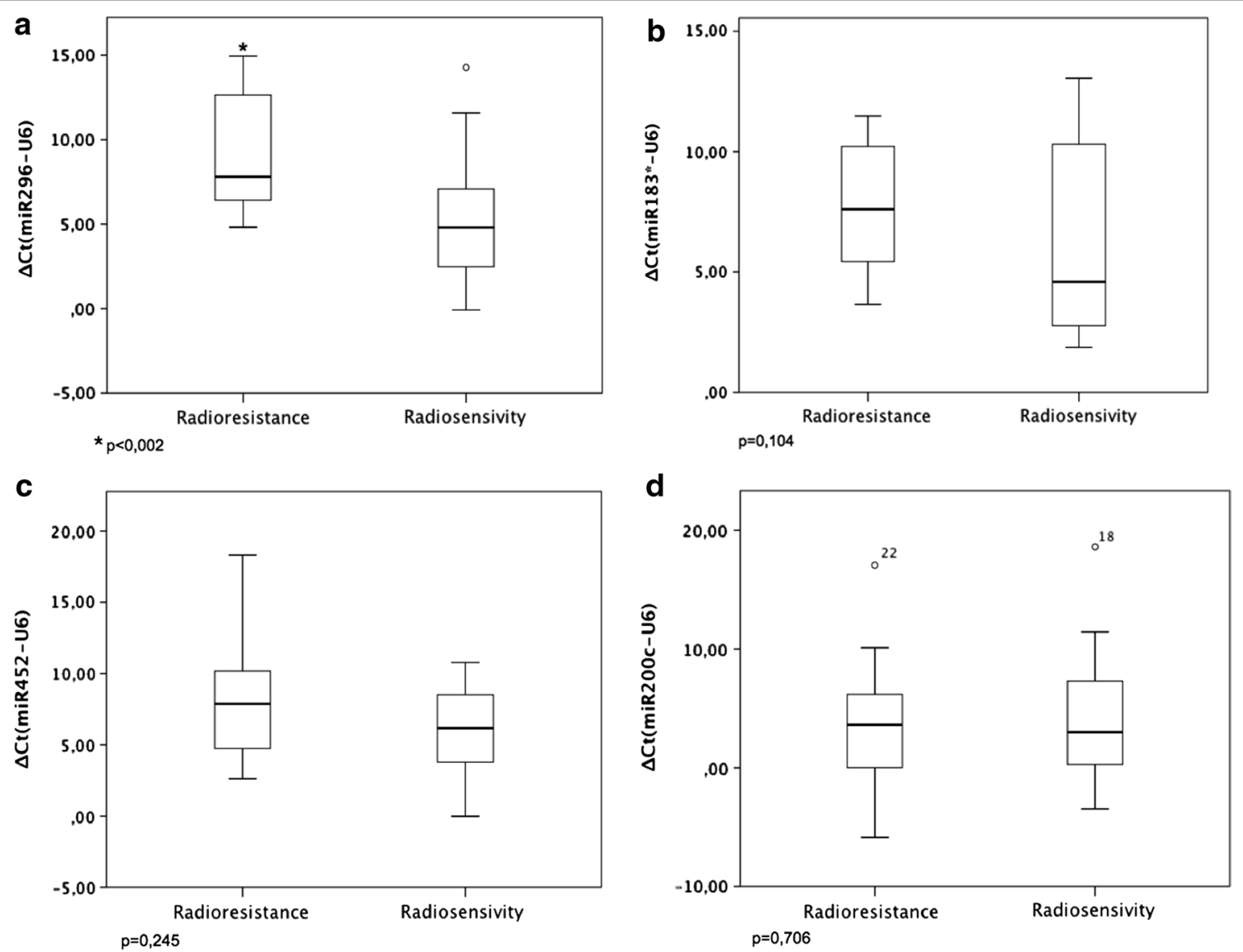

Fig. 1 Comparison between radioresistant and radiosensitive groups relative to expression level (Mann-Whitney test): a miR-296-5p, b miR-183, c miR-452, d miR-200c.
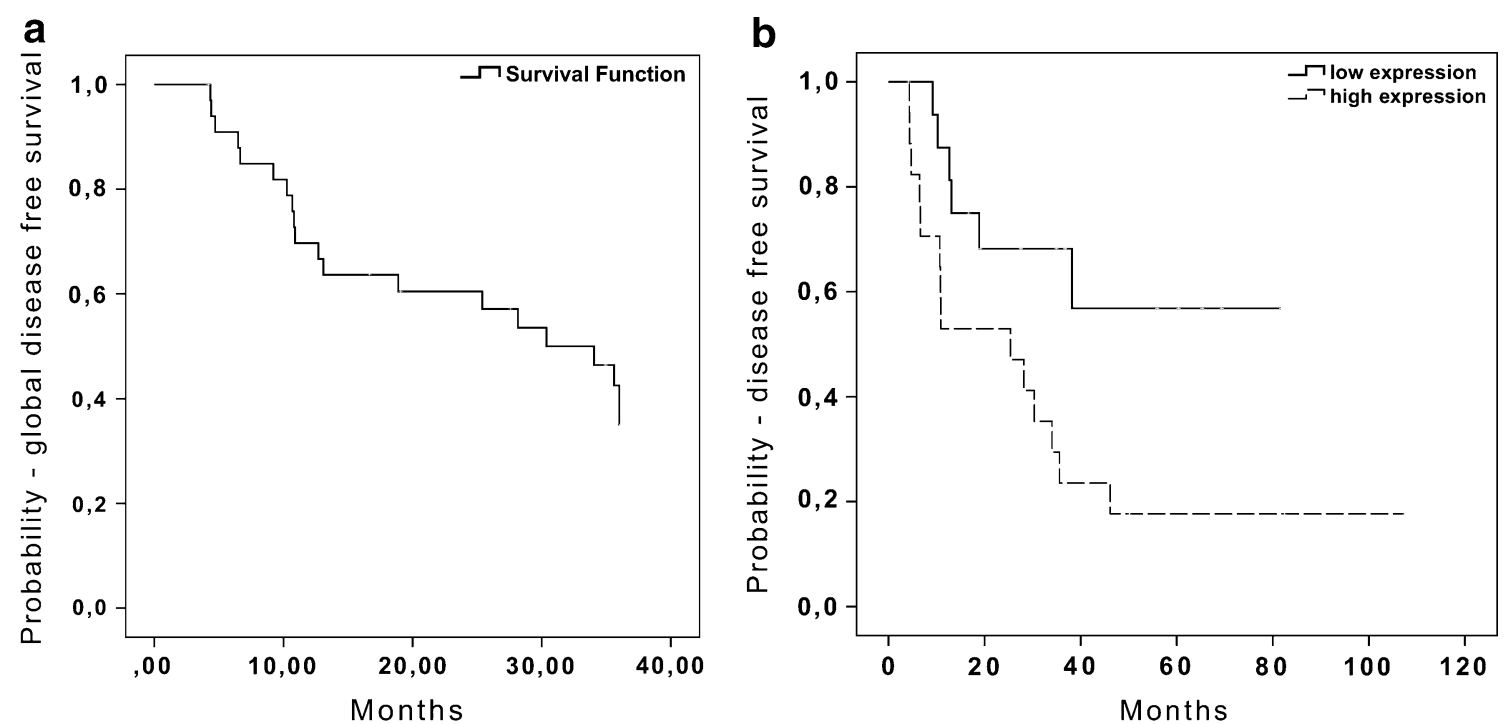

Fig. 2 Survival curves for 34 laryngeal squamous cell carcinoma patients. a 3-years disease-free survival for all patients. b 3-years disease-free survival rate according to miR-296-5p expression level in LSCC samples. 
early-stage laryngeal cancers fails in one third of the patients treated with definitive radiotherapy, we sought to identify miRNA-based markers which expression profile could discriminate between radiosensitive patients, who could actually benefit from this treatment approach, and radioresistant ones.

Cancer stem cells are known to play an important roll in radiation resistance and this important biological characteristic may be mediated through miRNA regulation [35, 36]. Huang et al. conducted a study in which laryngeal cancer stem cells were successfully isolated and than irradiated. The miRNA expression analyzes were performed before and after radiation and 70 miRNA were found to be differentially expressed. This experiment in cell lines demonstrated that laryngeal stem cells have different behavior in response to radiation, so tumor radioresistance observed in clinical practice may be intermediated by laryngeal stem cells through miRNA disorders [37]. The better understanding of miRNAs is essential for early interventions and better clinical results.

Of note, other studies have indicated miRNAs as important mediators of radioresistance in human tumors without focusing in stem cells, such as non-Hodgkin's lymphoma, oral squamous cell carcinoma, esophageal and lung cancers. Wu et al. (2012) showed that miR-148b is able to enhance radiosensitivity of non-Hodgkin's lymphoma cells by promoting radiation-induced apoptosis [38]. As such, the involvement of miRNAs in the radiation resistance of esophageal cancer cell was established by $\mathrm{Su}$ et al. [39]. Shiiba et al. demonstrated that down-regulated miR-125b expression was associated with radioresistance mechanisms in oral squamous cell carcinoma and they suggested that the control of the expression or activity of miR-125b might contribute to overcoming radioresistance in this oral malignancy [40]. Oh et al. found overexpression of miR-let-7a in radiosensitive A549 lung cancer cells in a miRNA profile study [41], while Grosso et al. showed that lung cancer cells expressing miR-210 exhibit a radioresistance similar to that found in hypoxic control cells [42]. According to this study, miR-210 stable expression mimics hypoxia-induced metabolic changes associated with a slight but significant stabilization of HIF- $1 \alpha$, and this information, combined with a strong reduction of radioresistance following HIF-1 silencing, reinforces the central role of HIF-1 in the resistance to radiotherapy. The molecular mechanism responsible for this radioresistance is not fully understood and is likely mediated by a complex network of miR-210 targets involved in a wide set of biological functions, including cell cycle control, survival, DNA repair and cell metabolism.

Our study was the first to evaluate the miRNA expression profile of LSCC and found miR-296-5p up-regulated in radioresistant patients and this high expression significantly correlates with tumor recurrence. The microRNA miR-296-5p is involved in many physiological and pathologic processes, such as angiogenesis, insulin metabolism, tumorigenesis and fetal alcohol syndrome [43-45]. Using a miRNA microarray approach to analyze the miRNA expression profile in esophageal cancer, Hong et al. showed that miR-296-5p might mediate drug resistance at least in part through regulation of MDR1 and apoptosis [46]. Furthermore, these authors also demonstrated that esophageal tumors with high expression of miR-296-5p had a worse prognosis compared with those with low expression. Along the same line, our findings also correlated the high expression of miR-296-5p with worse recurrence-free survival for early-stage LSCC patients.

The possibility of evaluating small fragments from early laryngeal carcinoma biopsies to predict the response to definitive radiotherapy is relevant given the difficulty of differentiating non-responder patients at high risk of relapse that should receive a more aggressive treatment, obviating potentially hazardous overtreatment in the group with a presumed favorable outcome. Based in the results present here, we may speculate that the expression of miR-296-5p in biopsy of laryngeal cancers could be a helpful biomarker to identify subjects resistant to radiotherapy. It is worth mentioning that the present study has some limitations. Remarkably, the patient number was limited. Therefore, further validation of these results requires studies with larger patient groups, which will allow the determination of cutoff values to easily classify the miR-296-5p expression level as "high" or "low". So, only after the establishment of these cutoffs and by achieving a good predictive negative value, this molecular approach could constitute a valuable tool to predict the radiotherapy response in a biopsy of the primary tumor, helping the clinicians in the adoption of the most effective treatment for early LSCC patients.

\section{Conclusions}

In the present study, the only one to our knowledge exploring the prognostic role of miRNAs in early laryngeal cancer in radiosensitive and radioresistant patients, we have shown that high expression level of miR296-5p is an adverse prognostic factor for recurrence in patients with early stage LSCC treated with definitive radiotherapy. Moreover, the evaluation of the miR296-5p expression could help clinicians to discriminate among LSCC patients who will take benefit from radiotherapy and who should take surgery as the first curative attempt. 


\section{Additional files}

Additional file 1: Table S1. Raw data of the expression level of 667 human microRNAs in LSCC samples according to the TaqMan Human MicroRNA Array assays.

Additional file 2: Table S2. Expression data of the four selected microRNAs in the 34 LSCC samples included in the study. Ct values represent the average of three technical replicates.

\section{Authors' contributions}

DCCM carried out the molecular biology studies, collected clinical data, analyzed data and performed the statistical analysis. CSN, have done histological evaluations. ACC helped in the molecular biology studies, data analyzes and helped to draft the manuscript. AFE analyzed PCR-array data. MAH helped in the molecular biology studies, data analyzes. ALC and ALV conceived the study, participated in its design and coordination and helped to draft the manuscript. All authors read and approved the final manuscript.

\section{Author details}

${ }^{1}$ Laboratory of Molecular Cancer Biology, Department of Biological Sciences, Federal University of São Paulo, Rua Pedro de Toledo, 669-11 $1^{\circ}$ andar, São Paulo, SP 04039-032, Brazil. ${ }^{2}$ Molecular Oncology Research Center, Barretos Cancer Hospital, Rua Antenor Duarte Vilela, 1331, Barretos, SP 14784-400, Brazil. ${ }^{3}$ Department of Head and Neck Surgery, Barretos Cancer Hospital, Rua Antenor Duarte Villela, 1331, Barretos 14784-400, Brazil. ${ }^{4}$ Department of Pathology, Barretos Cancer Hospital, Rua Antenor Duarte Villela, 1331, Barretos 14784-400, Brazil. ${ }^{5}$ Cancer and Stem Cell Biology Program, Duke-NUS Graduate Medical School, Singapore, Singapore.

\section{Acknowledgements}

This study was funded by São Paulo Research Foundation (FAPESP), Grant \# 2012/14837-7.

\section{Compliance with ethical guidelines}

\section{Competing interests}

The authors declare that they have no competing interests.

Received: 8 March 2015 Accepted: 28 July 2015

Published online: 12 August 2015

\section{References}

1. Ferlay J, Shin H, Bray F, Forman D, Mathers C, Parkin D (2010) Estimates of worldwide burden of cancer in 2008. Int J Cancer 2010:2893-2917

2. Barnes L, Eveson JW, Reichart P, Sidransky D (2005) Pathology and genetics of head and neck tumours. IARC Press, Lyon

3. Pfister D, Laurie S, Weinstein G, Mendenhall WM, Adelstein D, Ang K et al (2006) American Society of Clinical Oncology Clinical Practice Guideline for the use of larynx-preservation strategies in the treatment of laryngeal cancer. J Clin Oncol 24(22):3693-3704

4. American Society of Clinical O, Pfister DG, Laurie SA, Weinstein GS, Mendenhall WM, Adelstein DJ et al (2006) American Society of Clinical Oncology clinical practice guideline for the use of larynx-preservation strategies in the treatment of laryngeal cancer. J Clin Oncol 24:3693-3704

5. Cosetti M, Yu G-P, Schantz SP (2008) Five-year survival rates and time trends of laryngeal cancer in the US population. Arch Otolaryngol Head Neck Surg 134:370-379

6. Hera BHSC, Mdur ROJA, Orris CHGM, Irwan JEMK, Endenhall WIMM (2010) T1N0 and T2N0 Squamous cell carcinoma of the glottic larynx treated with definitive radiotherapy. Radiat Oncol 78:461-466

7. Aarts MCJ, Rovers MM, Grau C, Grolman W, van der Heijden GJM (2011) Salvage laryngectomy after primary radiotherapy: what are prognostic factors for the development of pharyngocutaneous fistulae? Otolaryngol Head Neck Surg Off J Am Acad Otolaryngol Head Neck Surg 144:5-9
8. Yamazaki H, Nishiyama K, Tanaka E, Koizumi M, Chatani M (2006) Radiotherapy for early glottic carcinoma (T1N0M0): results of prospective randomized study of radiation fraction size and overall treatment time. Int J Radiat Oncol Biol Phys 64:77-82

9. Begg AC (2012) Predicting recurrence after radiotherapy in head and neck cancer. Semin Radiat Oncol 22:108-118

10. Bese NS, Hendry J, Jeremic B (2007) Effects of prolongation of overall treatment time due to unplanned interruptions during radiotherapy of different tumor sites and practical methods for compensation. Int J Radiat Oncol Biol Phys 68:654-661

11. Rosenthal DI, Ang KK (2004) Altered radiation therapy fractionation, chemoradiation, and patient selection for the treatment of head and neck squamous carcinoma. Semin Radiat Oncol 2:153-166

12. Shaw AT, Yeap BY, Solomon BJ, Riely GJ, Gainor J, Engelman JA et al (2011) Effect of crizotinib on overall survival in patients with advanced nonsmall-cell lung cancer harbouring ALK gene rearrangement: a retrospective analysis. Lancet Oncol 12:1004-1012

13. Köhne $\mathrm{C}-\mathrm{H}$ (2010) How to integrate molecular targeted agents in the continuum of care. Ann Oncol 21(Suppl 7):vii134-vii139

14. Janne PA, Shaw AT, Pereira JR, Jeannin G, Vansteenkiste J, Barrios C et al (2013) Selumetinib plus docetaxel for KRAS-mutant advanced non-smallcell lung cancer: a randomised, multicentre, placebo-controlled, phase 2 study. Lancet Oncol 14:38-47

15. Camidge DR, Bang YJ, Kwak EL, lafrate AJ, Varella-Garcia M, Fox SB et al (2012) Activity and safety of crizotinib in patients with ALK-positive non-small-cell lung cancer: updated results from a phase 1 study. Lancet Oncol 13:1011-1019

16. Fleischhauer K, Shaw BE, Gooley T, Malkki M, Bardy P, Bignon JD et al (2012) Effect of T-cell-epitope matching at HLA-DPB1 in recipients of unrelated-donor haemopoietic-cell transplantation: a retrospective study. Lancet Oncol 13:366-374

17. He L, Hannon GJ (2004) MicroRNAs: small RNAs with a big role in gene regulation. Nat Rev Genet 5:522-531

18. Bartel DP, Lee R, Feinbaum R (2004) MicroRNAs: genomics, biogenesis, mechanism, and function genomics: the miRNA genes. Cell 116:281-297

19. Jin T, Lin HX, Lin H, Guo LB, Ge N, Cai XY et al (2012) Expression TGM2 and BNIP3 have prognostic significance in laryngeal cancer patients receiving surgery and postoperative radiotherapy: a retrospective study. J Transl Med 10:64

20. Kumar B, Cordell KG, D'Silva N, Prince ME, Adams ME, Fisher SG et al (2008) Expression of p53 and BCl-xL as predictive markers for larynx preservation in advanced laryngeal cancer. Arch Otolaryngol Head Neck Surg 134:363-369

21. Lu J, Getz G, Miska EA, Alvarez-Saavedra E, Lamb J, Peck D et al (2005) MicroRNA expression profiles classify human cancers. Nature 435:834-838

22. AJCC (1997) AJCC cancer staging manual, 5th edn. Springer, New York

23. Schmittgen TD, Livak KJ (2008) Analyzing real-time PCR data by the comparative CT method. Nat Protoc 3:1101-1108

24. Hui AB, Lenarduzzi M, Krushel T, Waldron L, Pintilie M, Shi W et al (2010) Comprehensive MicroRNA profiling for head and neck squamous cell carcinomas. Clin Cancer Res 16:1129-1139

25. Petrash HA, Philbrook A, Haburcak M, Barbagallo B, Francis MM (2013) ACR-12 ionotropic acetylcholine receptor complexes regulate inhibitory motor neuron activity in Caenorhabditis elegans. J Neurosci 33:5524-5532

26. Brown JS, Shaw RJ (2010) Reconstruction of the maxilla and midface: introducing a new classification. Lancet Oncol 11:1001-1008

27. Papagikos MA, Shaw EG, Stieber VW (2005) Lessons learned from randomised clinical trials in adult low grade glioma. Lancet Oncol 6:240-244

28. Schimming R, Reusch P, Kuschnierz J, Schmelzeisen R (2004) Angiogenic factors in squamous cell carcinoma of the oral cavity: do they have prognostic relevance? J Craniomaxillofac Surg 32:176-181

29. Ferracin M, Pedriali M, Veronese A, Zagatti B, Gafa R, Magri E et al (2011) MicroRNA profiling for the identification of cancers with unknown primary tissue-of-origin. J Pathol 225:43-53

30. Childs G, Fazzari M, Kung G, Kawachi N, Brandwein-Gensler M, McLemore $M$ et al (2009) Low-level expression of microRNAs let-7d and miR-205 are prognostic markers of head and neck squamous cell carcinoma. Am J Pathol 174:736-745 
31. Caltabiano R, Barbagallo GM, Castaing M, Cassenti A, Senetta R, Cassoni P et al (2013) Prognostic value of EGFR expression in de novo and progressed atypical and anaplastic meningiomas: an immunohistochemical and fluorescence in situ hybridization pilot study. J Neurosurg Sci 57:139-151

32. Fletcher AM, Heaford AC, Trask DK (2008) Detection of metastatic head and neck squamous cell carcinoma using the relative expression of miR205. Oncology 1:202-208

33. Ayaz L, Gorur A, Yaroglu HY, Ozcan C, Tamer L (2013) Differential expression of microRNAs in plasma of patients with laryngeal squamous cell carcinoma: potential early-detection markers for laryngeal squamous cell carcinoma. J Cancer Res Clin Oncol 139:1499-1506

34. Cao P, Zhou L, Zhang J, Zheng F, Wang H, Ma D et al (2013) Comprehensive expression profiling of microRNAs in laryngeal squamous cell carcinoma. Head Neck 35(5):720-728

35. Ghotra VP, Puigvert JC, Danen EH (2009) The cancer stem cell microenvironment and anti-cancer therapy. Int J Radiat Biol 85:955-962

36. Hambardzumyan D, Squatrito M, Holland EC (2006) Radiation resistance and stem-like cells in brain tumors. Cancer Cell 10:454-456

37. Huang CX, Zhu Y, Duan GL, Yao JF, Li ZY, Li D et al (2013) Screening for MiRNAs related to laryngeal squamous carcinoma stem cell radiation. Asian Pac J Cancer Prev 14:4533-4537

38. Maduri R, Barbagallo G, lofrida G, Signorelli M, Signorelli F (2013) Regression of Benedikt's syndrome after single-stage removal of mesencephalic cavernoma and temporal meningioma: a case report. Clin Neurol Neurosurg 115:748-750
39. Su H, Jin X, Zhang $X$, Xue S, Deng $X$, Shen L et al (2014) Identification of microRNAs involved in the radioresistance of esophageal cancer cells. Cell Biol Int 38:318-325

40. Adesso L, Calabretta S, Barbagallo F, Capurso G, Pilozzi E, Geremia R et al (2013) Gemcitabine triggers a pro-survival response in pancreatic cancer cells through activation of the MNK2/elF4E pathway. Oncogene 32:2848-2857

41. Oh JS, Kim JJ, Byun JY, Kim IA (2010) Lin28-let7 modulates radiosensitivity of human cancer cells with activation of K-Ras. Int J Radiat Oncol Biol Phys 76:5-8

42. Grosso S, Doyen J, Parks SK, Bertero T, Paye A, Cardinaud B et al (2013) MiR-210 promotes a hypoxic phenotype and increases radioresistance in human lung cancer cell lines. Cell Death Dis 4:e544

43. Wurdinger T, Tannous BA, Saydam O, Skog J, Grau S, Soutschek J et al (2008) miR-296 regulates growth factor receptor overexpression in angiogenic endothelial cells. Cancer Cell 14:382-393

44. Wang S, Olson EN (2009) AngiomiRs - key regulators of angiogenesis. Curr Opin Genet Dev 19:205-211

45. Barbagallo D, Piro S, Condorelli AG, Mascali LG, Urbano F, Parrinello N et al (2013) miR-296-3p, miR-298-5p and their downstream networks are causally involved in the higher resistance of mammalian pancreatic alpha cells to cytokine-induced apoptosis as compared to beta cells. BMC Genom 14:62

46. Hong L, Han Y, Zhang H, Li M, Gong T, Sun L et al (2010) The prognostic and chemotherapeutic value of miR-296 in esophageal squamous cell carcinoma. Ann Surg 251:1056-1063

\section{Submit your next manuscript to BioMed Central and take full advantage of:}

- Convenient online submission

- Thorough peer review

- No space constraints or color figure charges

- Immediate publication on acceptance

- Inclusion in PubMed, CAS, Scopus and Google Scholar

- Research which is freely available for redistribution

Submit your manuscript at

www.biomedcentral.com/submit

C Biomed Central 